\title{
Temperature-compensated fiber optic 3D shape sensor using femtosecond laser direct-written Bragg grating waveguides
}

K. K. C. Lee, A. Mariampillai, M. Haque, B. A. Standish, V. X. D. Yang, et al.

K. K. C. Lee, A. Mariampillai, M. Haque, B. A. Standish, V. X. D. Yang, P. R. Herman, "Temperature-compensated fiber optic 3D shape sensor using femtosecond laser direct-written Bragg grating waveguides," Proc. SPIE 8972, Frontiers in Ultrafast Optics: Biomedical, Scientific, and Industrial Applications XIV, 897210 (7 March 2014); doi: 10.1117/12.2042264 


\title{
Temperature-compensated fiber-optic 3D shape sensor using femtosecond laser direct-written Bragg grating waveguides
}

\author{
K.K.C. Lee ${ }^{*} a, b$, A. Mariampillai ${ }^{c}$, M. Haque $^{a}$, B.A. Standish ${ }^{b}$, V.X.D. Yang ${ }^{a, b}, d, e$, and P.R. \\ Herman $^{a}$ \\ ${ }^{a}$ Institute for Optical Sciences, and the Department of Electrical and Computer Engineering, \\ University of Toronto, Canada; \\ ${ }^{b}$ Department of Electrical and Computer Engineering, Ryerson University, Toronto, Canada; \\ ${ }^{c} 7 \mathrm{D}$ Surgical Inc., Toronto, Canada; \\ ${ }^{d}$ Division of Neurosurgery, University of Toronto, Canada; \\ ${ }^{e}$ Division of Neurosurgery, Sunnybrook Health Sciences Center, Toronto, Canada
}

\begin{abstract}
A fiber-optic 3D shape, position and temperature sensor is demonstrated with femtosecond laser direct-written optical and Bragg grating waveguides that were distributed axially and radially inside a single coreless optical fiber. Efficient light coupling between the laser-written optical circuit elements and a standard single-mode optical fiber was obtained by 3D laser writing of a $1 \times 3$ directional coupler and fusion splicing. Coupler optimization by real-time monitoring of Bragg grating strengths is discussed. Simultaneous interrogation of nine Bragg gratings is presented through a single waveguide port to follow the Bragg wavelength shifts and thereby infer shape and temperature profile along the fiber length.
\end{abstract}

Keywords: Bragg grating waveguides, femtosecond direct laser writing, fiber cladding photonics, fiber optics sensors

\section{INTRODUCTION}

Conventional uniform fiber Bragg gratings (FBGs) are conveniently fabricated with holographic or phase mask interference method in the waveguiding core of standard single-mode optical fibers (SMFs). ${ }^{1}$ Owing to their position at the fiber's center (i.e. neutral axis), these FBGs are naturally insensitive to bend-induced strain and hence cannot be used to sense the fiber shape. To overcome this limitation, Duncan et al. leveraged the eccentric waveguide cores of a multicore fiber (MCF) and demonstrated fiber shape sensing with a dense array of axially and radially distributed FBGs. ${ }^{2}$ However, simultaneous interrogation of the multiple waveguide cores remain a major packaging challenge in this approach, demanding high precision and cumbersome alignment to multiple tapered SMFs.

Femtosecond direct laser writing in transparent materail exploits nonlinear light-material interaction to induce highly localized and positive index modification. Optical components such as uniform optical waveguides, Bragg grating waveguides (BGWs), and directional couplers have been demonstrated in fused silica and are the key building blocks of 3D optical circuits. In particular, direct laser writing of a 3D directional coupler inside optical fibers offers the novel opportunity to couple light between the radially and axially distributed BGW sensor elements and meet with a center waveguide core to overcome the major packaging challenge with MCF-type sensors as we reported recently. ${ }^{3}$

In this report, we describe the fiber-optic 3D shape, position, and temperature sensor that was formed by direct laser writing of a network of radially and axially distributed BGWs in a coreless optical fiber, which coupled conveniently to a SMF by fusion splicing. A laser-written $1 \times 3$ directional coupler was the key element

Further author information:

E-mail: keneth.lee@mail.utoronto.ca

Full address: The Edward S. Rogers Sr. Department of Electrical and Computer Engineering, University of Toronto, 10 King's College Rd., Toronto, Ontario, M5S 3G4, Canada

Frontiers in Ultrafast Optics: Biomedical, Scientific, and Industrial Applications XIV, edited by Alexander Heisterkamp,

Peter R. Herman, Michel Meunier, Stefan Nolte, Proc. of SPIE Vol. 8972, 897210

(C) 2014 SPIE · CCC code: 0277-786X/14/\$18 - doi: 10.1117/12.2042264

Proc. of SPIE Vol. $8972897210-1$ 
to distribute light into the center and off-center fiber positions for unambiguous temperature and strain measurement, respectively. The coupler also enabled simultaneous monitoring of all nine BGWs through a single waevguide port of the SMF, overcoming the packaging challenge of the MCF approach. Fiber shape sensing to a $77 \mathrm{~mm}$ bend radius and temperature sensing in the 24 to $100{ }^{\circ} \mathrm{C}$ range are demonstrated.

\section{SENSOR DESIGN AND FABRICATION}

Figure 1(a) is a schematic of the shape sensor showing all the optical components that were direct laser written inside the coreless optical fiber. The coreless fiber has a cladding diameter of $125 \mathrm{\mu m}$ and was fusion spliced to a standard SMF-28 optical fiber prior to laser processing. The optical components included a center laserwritten waveguide to couple efficiently to the SMF core as shown by the microscope image of the spliced plane in Fig. 1(b). Two additinal parallel waveguides located $9 \mu \mathrm{m}$ away from the fiber center (Fig. 1(c)) formed the $1 \times 3$ directional coupler to split the light among all three waveguides after $0.5 \mathrm{~mm}$ coupling length. Two S-bend waveguides, each consisted of two opposing and symmetric arcs with constant radius of $40 \mathrm{~mm}$, brought the off-center waveguides to their final radial position of $40 \mu \mathrm{m}$ as shown in fiber's cross-sectional image of Fig. 1(d). Lastly, three $1 \mathrm{~cm}$ long BGWs spaced $1 \mathrm{~cm}$ edge-to-edge apart were laser written in tandem with each straight uniform waveguide, yielding a total of nine Bragg wavelengths from $\lambda_{1}=1280 \mathrm{~nm}$ to $\lambda_{9}=1320 \mathrm{~nm}$, with $5 \mathrm{~nm}$ increments. This short to long wavelength ordering ensured that the spectrum of cladding-mode-coupled light from an upstream BGW would not coincide with the resonance of a downstream BGW.4 The BGWs in the two orthogonal off-center waveguides were used to infer the fiber's directional curvature while the BGWs in the fiber center were used to measure temperature and provide temperature compensation for the radially adjacent BGWs.

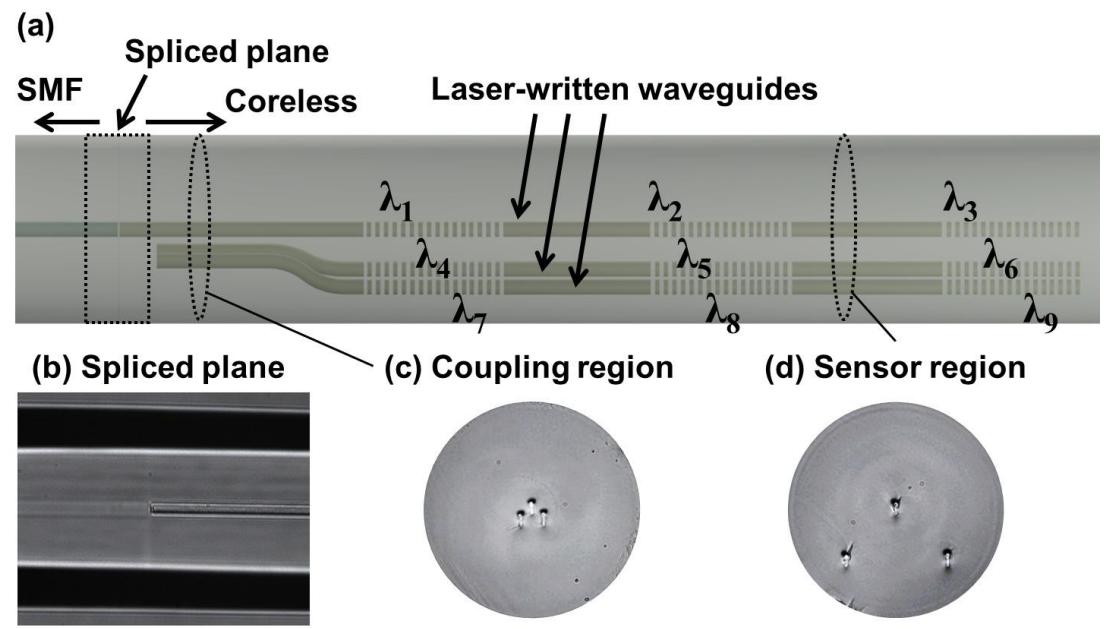

Figure 1. Schematic (a) of the fiber-optic shape sensor showing a side-view of the coreless fiber fusion spliced to a SMF and the arrangement of optical components within. Microscope image (b) of the spliced plane showing where the laser-written waveguide is aligned to the SMF core. Cross-sectional images of the coupling (c) and sesnor (d) region, respectively, showing the radial arrangement of the waveguides. The fiber diameter is $125 \mu \mathrm{m}$.

All optical components were written in a single exposure step with a femtosecond fiber laser $(230 \mathrm{fs}, 522 \mathrm{~nm}$, $1 \mathrm{MHz}$ ) following the procedure previously presented by Grenier et al. ${ }^{5}$ The laser was focsued into the fiber with a $100 \times, 1.25 \mathrm{NA}$ oil immersion objective lens to avoid the strong astigmatic and spherical aberration of the cylindrical fiber geometry. A laser exposure of $71 \mathrm{~nJ}$ pulse energy and $0.1 \mathrm{~mm} / \mathrm{s}$ scan speed produced optimal waveguides with $0.5 \mathrm{~dB} / \mathrm{cm}$ propagation loss and $7.5 \mu \mathrm{m} \times 6.5 \mu \mathrm{m}$ mode field diameter. Periodicity and therefore the desired Bragg resonance, $\lambda_{i}$, of each BGW was controlled by the frequecy supplied to an acousto-optic modulator for exposure at $60 \%$ duty cycle as previously described by Zhang et al. ${ }^{6}$ 


\section{$2.11 \times 3$ Directional Coupler Optimization}

A balanced coupling ratio in the $1 \times 3$ directional coupler was desired to ensure that all Bragg resonances could be detected above the noise floor. A parameter scan of various laser exposure and coupler geometry was first applied in planar bulk glass to find the optimal coupling separation and length to obtain a balanced coupling in the isosceles right triangle configuration as shown in Fig. 2(a) with waveguide ports labeled from 1 to 3 . Vertical and horizontal polarized light were individually lauched from one end of port 3 , and the power was measured at each of the ports at the other end of the directional coupler. The coupling ratio is defined as the ratio between the measured power at one output port and the sum of power from the three exit ports. Figure 2(c) and (d) shows the coupling ratio measured as function of coupling length ranging from 0 to $1.6 \mathrm{~mm}$ for vertical and horizontal polarization respectively at $1300 \mathrm{~nm}$ wavelength and a coupling separation of $9 \mu \mathrm{m}$. As coupling length increased from 0 to $1 \mathrm{~mm}$, the power split almost equally into port 1 and 2 , while the power in port 3 decreased. A coupling length of $0.4-0.5 \mathrm{~mm}$ and $0.6-0.8 \mathrm{~mm}$ yielded approximately $1 / 3$ power splitting ratio for vertical and horizontal polarization respectively. Using a coupling separation and length of $9 \mu \mathrm{m}$ and $450 \mu \mathrm{m}$, the coupling ratio is plotted against wavelength in Fig. 2(b) showing an expected wavelength dependency of optical directional couplers. A slight birefringence in the coupling ratio arises from asymmetric stresses induced in the glass during waveguide writing. Nonetheless, the coupling ratio remained sufficiently constant $( \pm 5 \%)$ within the wavelength range of interest (1275-1325 nm) to accommodate nine Bragg gratings.

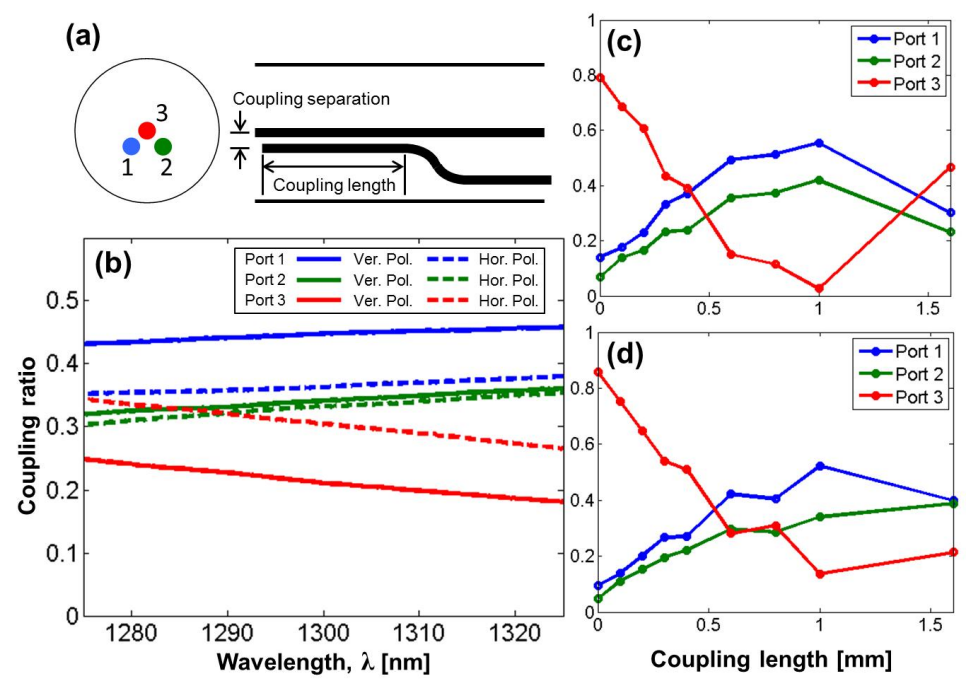

Figure 2. Schematic (a) of the $1 \times 3$ directional coupler with waveguides placed in an isosceles right triangle and ports labeled from 1-3. Measured coupling ratio versus wavelength (b) for coupling separation and length of $9 \mu \mathrm{m}$ and $450 \mu \mathrm{m}$ respectively. Measured coupling ratio versus coupling length for vertical (c) and horizontal (d) polarization respectively.

When laser writing the directional coupler in the coreless fiber, the coupling length was further optimized by monitoring the relative Bragg grating strengths with a high speed spectrometer. Figure 3(a) shows the straightforward experimental setup where the broadband light source with 1275 to $1345 \mathrm{~nm}$ spectrum was launched through an optical circulator into the SMF and the reflected Bragg wavelengths were simultaneously interrogated with the spectrometer at $1 \mathrm{kHz}$ sampling rate during the waveguide writing process. Each waveguide of the directional coupler was laser written in sequence starting with the center waveguide followed by the two off-center waveguides. As the coupling length was lengthened by the laser at a rate of $0.1 \mathrm{~mm} / \mathrm{s}$ (as indicated by the dotted black arrow in Fig. 3(a)), real-time capture of Bragg resonance strength provided a direct feedback of the coupling ratio. The writing was terminated when the desired balanced coupling ratio was obtained. The resultant reflection spectrum is shown in Fig. 3(b) recorded when the fiber was straight and unstrained at room temperature. The Bragg resonances are numbered in accordance with their corresponding locatoins shown in Fig. 1(a). The slight blue shift of $\sim 1 \mathrm{~nm}$ observed in all the Bragg wavelengths resulted from tension release of the fiber after laser processing. The logarithmic falloff of $\sim 5 \mathrm{~dB}$ between the downstream BGWs, $\lambda_{3}, \lambda_{6}, \lambda_{9}$, 
with respect to their corresponding upstream BGWs, $\lambda_{1}, \lambda_{4}, \lambda_{7}$, inferred a BGW propagation loss of $0.7 \mathrm{~dB} / \mathrm{cm}$ which is slightly larger than the $0.5 \mathrm{~dB} / \mathrm{cm}$ loss for a uniform waveguide as expected due to higher radiation loss in a less uniform BGW.
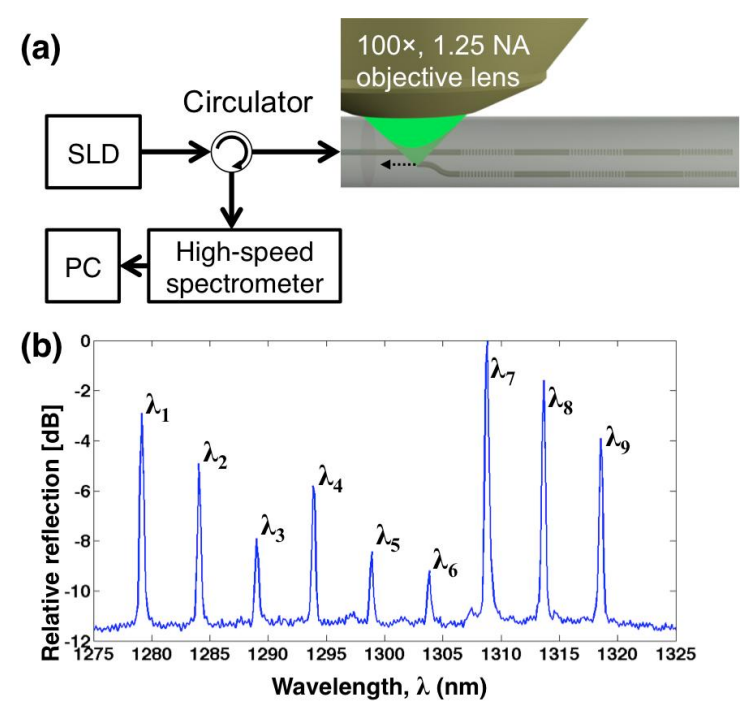

Figure 3. Schematic (a) of the experimental setup that enabled real-time characterization of the directional coupler during fabrication. The black dotted arrow shows the laser writing direction toward the SMF. SLD: superluminescent diode, PC: personal computer. Reflection spectrum (b) recorded with the spectrometer after device fabrication, simultaneously displaying all nine Bragg wavelengths that are numbered in accordance to their positions shown in Fig. 1(a).

\section{FIBER SHAPE SENSING}

The femtosecond laser-written Bragg grating wavegudies follow the conventional response of FBGs in SMFs, which relates the Bragg wavelength shift, $\Delta \lambda$, to strain, $\epsilon$, and temperature variation, $\Delta T$, as expressed by Eq. $(1){ }^{7}$

$$
\frac{\Delta \lambda}{\lambda}=\left(1-p_{e}\right) \epsilon+\left(\alpha+\frac{1}{n} \zeta\right) \Delta T
$$

Here, $p_{e}$ is the effective strain-optic constant, $\alpha=0.55 \times 10^{-6} \mathrm{~K}^{-1}$ is the thermal expansion coefficient for fused silica, ${ }^{1} n=1.447$ is the refractive index for fused silica near $1300 \mathrm{~nm}$ wavelength, ${ }^{8}$ and $\zeta$ is the thermo-optic coefficient. As previously measured in [3], strain sensitivity of $\Delta \lambda / \epsilon=1.06 \pm 0.06 \mathrm{pm} / \mathrm{\mu} \epsilon$ and thermal response of $\Delta \lambda / \Delta T=9.1 \pm 0.6 \mathrm{pm} /{ }^{\circ} \mathrm{C}$ inferred a strain-optic constant of $p_{e}=0.194$ and thermo-optic coefficient of $\zeta=9.5 \times 10^{-6} \mathrm{~K}^{-1}$, respectively.

In the absense of thermal gradients, the fiber shape was calculated from the Bragg wavelength shift of the six off-center BWGs monitored with the same setup shown in Fig. 3(a). The bend-induced strain, $\epsilon=d C$, was first converted to localized directional curvature, $C$, using the expected radial offset position of $d=40 \mu \mathrm{m}$ as designed for the off-center waveguides as seen in the fiber's cross-sectional image in Fig. 1(d). Cubic spline interpolation was used to esimate the curvature between axial BGW positions and a fixed start position (i.e. origin). To accomodate the large geometric deformation, the shape and position of the fiber was then determined from the continuously varying curvature function using the finite element method of co-rotational analysis, which discretized the fiber into curved rigid beam elements that were aligned tangentially at each node. ${ }^{9}$ Using said method, fiber shape sensing at room temperature is demostrated in Fig. 4 with four different calculated fiber shapes including the unstrained straight fiber position indicated by the black line and BGWs locations indicated by the green line segments. Figure $4(\mathrm{a})$ is a 3D view of the fiber shapes and (b-d) are three corresponding orthogonal views, $\mathrm{x}-\mathrm{y}, \mathrm{x}-\mathrm{z}$, and $\mathrm{y}-\mathrm{z}$, respectively. Overall, the fiber shape sensing could precisely follow the 
physical BGW displacements (green segments in Fig. 4) of up to $26 \mathrm{~mm}$ and bending radii as small as $77 \mathrm{~mm}$. A small positon error of $\sim 0.6 \mathrm{~mm}$ was observed in the proximity of the BGWs but this accuracy diminished far outside the distributed BGW sensing zone. ${ }^{3}$ Similarly, 3D fiber shape can be monitored in real-time at $1 \mathrm{kHz}$ sampling frequency with the high-speed spectrometer as fully explored in [3].
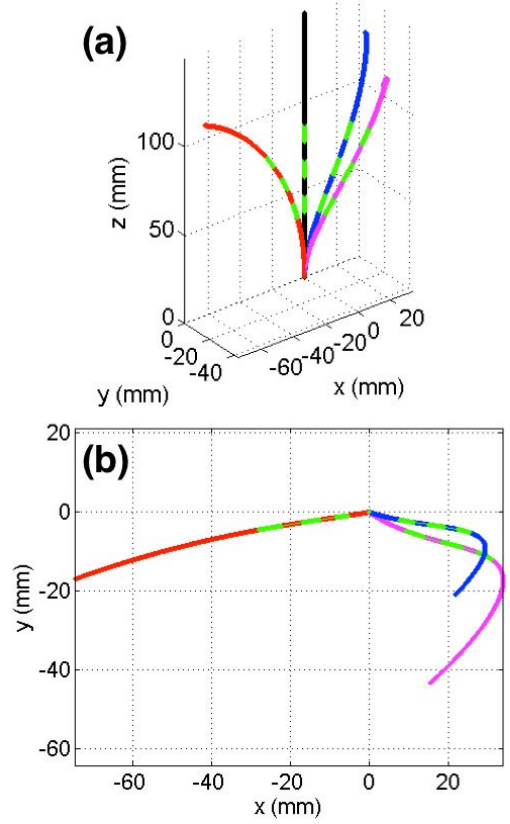
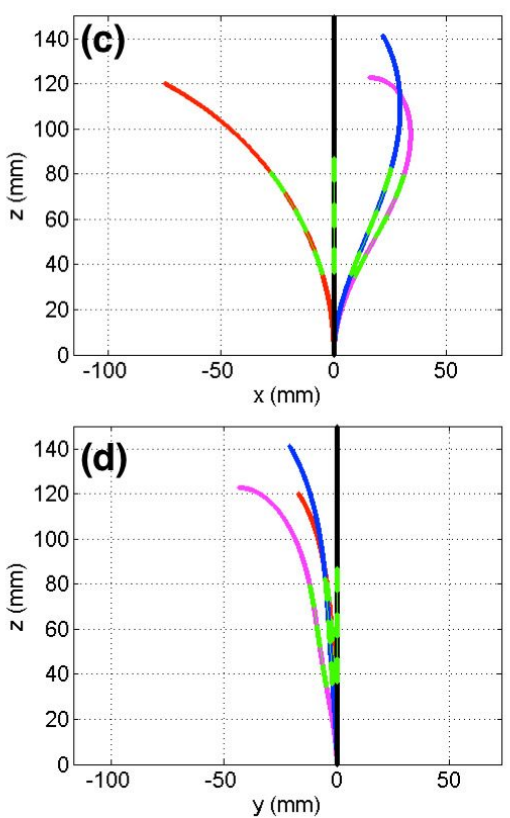

Figure 4. 3D plot (a) of four different fiber shapes inferred from wavelength shifts in a distributed BGW sensor undergoing bending strain. The black line indicates the unstrained straight fiber position while green segments highlight the BGWs locations in the fiber. Corresponding orthogonal views, $x-y(b), x-z(c)$, and $y-z(d)$, respectively.

In Fig. 5(a) the fiber was subjected to non-uniform bending and heating with a hot iron. In this case, temperature in the range of 24 to $100{ }^{\circ} \mathrm{C}$ was calculated from the wavelength shift of the center BGWs as they are naturally insensitive to bend-induced strain due to their position in the fiber's neutral axis. Moreover, temperature was linear interpolated in between each axially co-located BGW triplets. Assuming uniform temperature in the fiber's cross section, the fractional wavelenght shifts of the center and off-center BGWs were then temperature-corrected to provide the distributed fiber strain values, and thus offer the measure of the temperature gradient seen along the fiber in false color together with the fiber sensor shape. For comparison, the calculated fiber shape is also shown in Fig. 5(b) for when the fiber was bent to the same position at room temperature. Figure 5(c) shows the corresponding reflection spectrum for Fig. 5(a) and (b) in dashed and solid line, respectively, where the unstrained and room-temperature Bragg wavelengths are marked by the red lines and BGWs in each sensing arm (i.e. center and off-center) further labeled by a different color. As expected during fiber bending at room temperature, large wavelength shifts are observed in the off-center BGWs relative to the non-shifing center BGWs. In contrast, heating caused a shift in all the Bragg wavelengths in addition to the wavelength shift incurred by fiber bending.

\section{CONCLUSION}

A temperature-compensated fiber-optic 3D shape sensor was designed and fabricated by femtosecond laser direct writing of various optical components in a coreless optical fiber. The $3 \mathrm{D}$ formation of a $1 \times 3$ coupler was instrumental in facilitating efficient and nearly balanced light coupling and collection from the laser-written optical circuit elements for convenient interrogation through a fusion-spliced SMF. This approach offered advantages in facile and flexible fabrication in comparison with prior demonstrations of shape sensors that was based on a MCF. ${ }^{2}$ The geometry of the nine axially and radially distributed BGW sensors enabled decoupling of the Bragg 

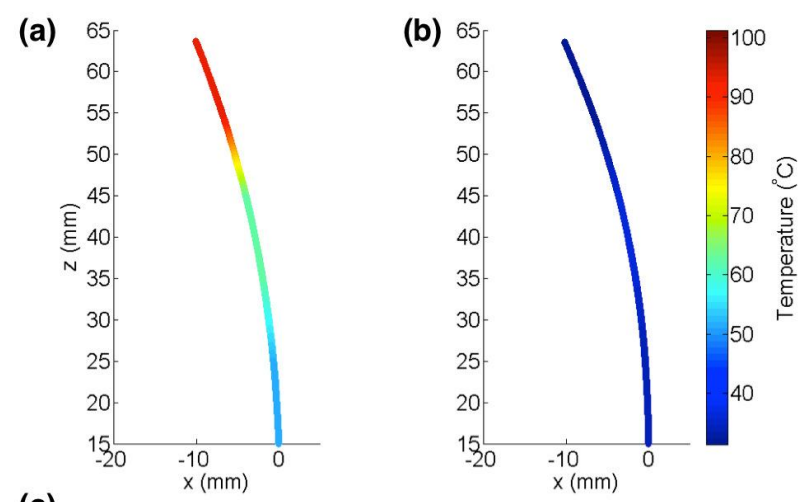

(c)

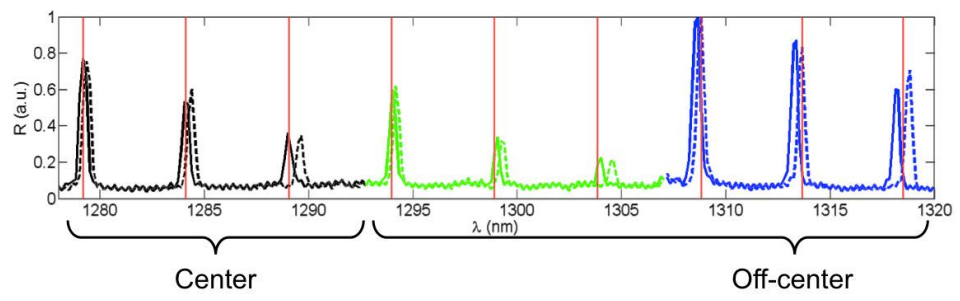

Figure 5. Fiber shape and temperature profiles $(\mathrm{a}-\mathrm{b})$ calculated from the Bragg wavelength shifts in reflection spectrum of nine BGWs in (c), where the red lines indicate the Bragg wavelengths of unstrained gratings at room temperature. Fiber bending under graded (a) and uniform (b) temperature.

wavelength shifts for simultaneous strain and temperature measurements on which to infer real-time shape and thermal profile along the fiber length. The overall compact size and high temperature tolerance make the present fiber sensor attractive for structural, industrial, reactor, and pipeline applications as well as for integration into biomedical and catheter devices.

\section{ACKNOWLEDGMENTS}

The authors gratefully acknowledge financial support from the Natural Sciences and Engineering Research Council of Canada and the Early Researcher Award (Ontario, Canada).

\section{REFERENCES}

[1] Kashyap, R., [Fiber Bragg Gratings], Academic Press (Oct. 2009).

[2] Duncan, R. G., Froggatt, M. E., Kreger, S. T., Seeley, R. J., Gifford, D. K., Sang, A. K., and Wolfe, M. S., "High-accuracy fiber-optic shape sensing," in [Proceedings of SPIE], (2007).

[3] Lee, K. K. C., Mariampillai, A., Haque, M., Standish, B. A., Yang, V. X. D., and Herman, P. R., "Temperature-compensated fiber-optic 3D shape sensor based on femtosecond laser direct-written Bragg grating waveguides," Optics express 21(20), 24076-24086 (2013).

[4] Martinez, A., Dubov, M., Khrushchev, I., and Bennion, I., "Direct writing of fibre Bragg gratings by femtosecond laser," Electronics Letters 40(19), 1170-1172 (2004).

[5] Grenier, J. R., Fernandes, L. A., Marques, P. V., Aitchison, J. S., and Herman, P. R., "Optical circuits in fiber cladding: Femtosecond laser-written bragg grating waveguides," in [CLEO:2011 - Fiber Devices (CMZ)], Optical Society of America, Baltimore (2011).

[6] Zhang, H., Eaton, S. M., and Herman, P. R., "Single-step writing of Bragg grating waveguides in fused silica with an externally modulated femtosecond fiber laser," Optics letters 32(17), 2559-2561 (2007).

[7] Hill, K. O. and Meltz, G., "Fiber Bragg Grating Technology Fundamentals and Overview," Lightwave Technology 15, 1263 (Aug. 1997). 
[8] Leviton, D. B. and Frey, B. J., "Temperature-dependent absolute refractive index measurements of synthetic fused silica," in [Proceedings of SPIE], (May 2006).

[9] Crisfield, M. A., "A consistent co-rotational formulation for non-linear, three-dimensional, beam-elements," Computer Methods in Applied Mechanics and Engineering 81, 131-150 (Aug. 1990).

Proc. of SPIE Vol. $8972897210-7$

Downloaded From: https://www.spiedigitallibrary.org/conference-proceedings-of-spie on 11/22/2018 Terms of Use: https://www.spiedigitallibrary.org/terms-of-use 